\title{
Quantitative analysis of colorectal mucosal lesions by autofluorescence endoscopy: Discrimination of carcinomas from other lesions
}

\author{
KEIKO ARITA $^{1}$, KEIICHI MITSUYAMA ${ }^{1}$, HIROSHI KAWANO ${ }^{1}$, SHIN HASEGAWA ${ }^{1}$, \\ YASUHIKO MAEYAMA $^{1}$, JUNYA MASUDA ${ }^{1}$, YOSHITO AKAGI ${ }^{2}$, YASUTOMO WATANABE ${ }^{1}$, \\ YOSHINOBU OKABE ${ }^{1}$, OSAMU TSURUTA ${ }^{1}$ and MICHIO SATA ${ }^{1}$ \\ ${ }^{1}$ Division of Gastroenterology, Department of Medicine; ${ }^{2}$ Department of Surgery, Kurume \\ University School of Medicine, Asahi-machi 67, Kurume 830-0011, Japan
}

Received January 18, 2011; Accepted February 28, 2011

DOI: $10.3892 /$ or.2011.1287

\begin{abstract}
A newly developed autofluorescence (AF) imaging technique was applied during colonoscopy in a clinical setting. This pilot study was conducted to evaluate the clinical feasibility of applying AF endoscopy for distinguishing colorectal lesions. A total of 54 colorectal mucosal lesions obtained from 43 patients who underwent both white-light and AF endoscopy and were treated by endoscopy or surgery were assessed. Of the lesions, 11 were hyperplastic polyps, 30 were adenomas and 13 were carcinomas. To quantify the $\mathrm{AF}$ intensity, a color-contrast index (CCI) was determined and evaluated in relation to the histology, size and shape of each lesion. CCI was significantly associated with the histology and size of the lesions, but not their shape. CCI increased as the malignant potential increased (in the order of hyperplastic polyps $\rightarrow$ adenomas $\rightarrow$ carcinomas), irrespective of the lesion size $(r=0.797, p<0.0001$ for size $>8 \mathrm{~mm} ; \mathrm{r}=0.622, \mathrm{p}=0.0045$ for size $>8 \mathrm{~mm}$ but $>15 \mathrm{~mm}$; $\mathrm{r}=0.644, \mathrm{p}=0.0071$ for size $>15 \mathrm{~mm}$ ). In each size group, CCI tended to be higher for carcinomas than for adenomas, and also higher for adenomas than for hyperplastic polyps. CCI allowed discrimination of adenomas/carcinomas from hyperplastic polyps with $95.3 \%$ sensitivity and $63.6 \%$ specificity (cut-off value, 14.5 ), and of colorectal carcinomas from adenomas with $84.6 \%$ sensitivity and $80.0 \%$ specificity (cut-off value, 28.0). These results suggest that the quantitative analysis of AF intensity using CCI is helpful to discriminate among different types of colorectal mucosal lesions, including carcinomas.
\end{abstract}

Correspondence to: Dr Keiichi Mitsuyama, Division of Gastroenterology, Department of Medicine, Kurume University School of Medicine, 67 Asahi-machi, Kurume 830-0011, Japan

E-mail: ibd@med.kurume-u.ac.jp

Key words: colorectal cancer, carcinoma, adenoma, hyperplastic polyp, autofluorescence

\section{Introduction}

Colorectal mucosal lesions are commonly encountered gastrointestinal tract lesions, and include neoplastic lesions (adenomas and carcinomas) and non-neoplastic lesions (hyperplastic polyps). Colonoscopy is the only technique currently available that offers the potential for detection and removal of mucosal lesions throughout the large intestine. Although accurate histological interpretation of mucosal lesions visualized by colonoscopy is essential for the subsequent clinical management of the patient, there is a substantial amount of misdiagnosis with this procedure, resulting in the possibility of inadequate treatment.

Currently, several techniques have been attempted to improve this limitation of conventional colonoscopy (1), including chromoendoscopy $(2,3)$ and narrow-band imaging $(4,5)$. Although these tools have good diagnostic accuracy for differentiating among colorectal lesions, they are rather subjective because they are based on visual identification of pit patterns and vascular patterns, respectively.

Autofluorescence (AF) endoscopy is a novel imaging technology in which endogenous fluorophores in the tissues are stimulated when the mucosa is illuminated by a shortwavelength light and a fluorescent light of a longer wavelength (6-10). Recently, several groups have reported on the usefulness of AF endoscopy in the differential diagnosis of colorectal mucosal lesions, suggesting that AF endoscopy is useful for distinguishing adenomas from hyperplastic polyps (11-15). However, little is known about the role of AF endoscopy in the diagnosis of colorectal carcinomas. In the present study, we investigated the efficacy of AF endoscopy in distinguishing between neoplastic and non-neoplastic lesions, and among the neoplastic lesions, between adenomas and carcinomas.

\section{Patients and methods}

Patient selection and study design. A total of 43 patients (36 men and 18 women; mean age, 62.9 years) who underwent total colonoscopy with AF as well as white-light endoscopy at Kurume University Hospital between September 2005 
and August 2008, who had one or more colorectal mucosal lesions that satisfied all of the following inclusion criteria for the study, were enrolled in this study: i) lesions for which the prototype AF endoscopy was possible; ii) lesions which were subsequently managed by endoscopic or surgical resection; iii) lesions in which post-operative histopathological evaluation was possible; and iv) lesions which could be excluded as being advanced colorectal carcinomas.

Ethical considerations. The Ethics Committee of our hospital approved the study protocol and written informed consent was obtained from each of the study participants.

Endoscopic procedure. All patients underwent preparation for colonoscopy by ingesting 2 liters of polyethylene glycol-electrolyte solution on the morning of the procedure. Scopolamine butylbromide $(10 \mathrm{mg}$ ) was administered intravenously to avoid bowel movements prior to the examination in those patients in whom this agent was not contraindicated. Colonoscopic examinations were performed using white-light and AF imaging of the large intestine by experienced colonoscopists.

Autofluorescence imaging system. The AF imaging system (Olympus Medical Systems, Tokyo, Japan) used in this study consisted of a light-source system (CLV-260SL), a processor (CV-260SL), a liquid-crystal display monitor, and a specialized video endoscope for AF detection (CF-FH260AZI). This system allowed switching between white-light endoscopy to AF endoscopy by the press of a button in the control head of the endoscope. Furthermore, this AF imaging videoendoscope is equipped with an accessory channel having an internal diameter of $3.2 \mathrm{~mm}$. The outer diameter of the distal tip of this AF imaging videoscope is $14.8 \mathrm{~mm}$, and the scope also has the function of variable stiffness and magnification (up to x75 in conventional imaging).

In this system, white light emitted from a $300-\mathrm{W}$ xenon lamp is separated by a rotary filter into an excitation light with a wavelength range of 390-470 $\mathrm{nm}$ and a green light with a wavelength of 540-560 $\mathrm{nm}$. The mucosa is irradiated sequentially with these fractionated lights during the observation period. A barrier filter to remove the reflected excitation light is set in front of a monochrome charge-coupled device. Light of $500-630 \mathrm{~nm}$ wavelength is selectively detected from both the AF and reflected green light. A false color image is produced by allocating the detected and amplified AF signals to the green $(\mathrm{G})$ channel, and the reflected signal of the green light to the red (R) and blue (B) channels in the ratio of 1 to 0.5 .

Autofluorescence intensity analysis. Endoscopic images were automatically saved in an 8-bit bitmap format on a computer besides the videoendoscopy system. The $R, G$ and $B$ values in the standard (s)RGB color space were measured using coloranalysis software. The values are defined by the International Electrotechnical Commission and run from 0 through 255 in proportion to the light energy that enters the charge-coupled device. The color-analysis software was developed by Olympus Medical Systems, Tokyo, Japan, and preinstalled on the same computer. By using this software, we could analyze the $R, G$ and $B$ values and their inverse $\gamma$-corrected values for the sampled areas. Color-tone sampling was performed on broad areas of the lesions. The mean $\mathrm{R}, \mathrm{G}$ and $\mathrm{B}$ values were applied for the analysis. Due to individual variations in the AF intensity, the AF intensity of any identified lesion was compared with the intensity of the adjacent normal area in each individual, and the corrected value, termed color-contrast index (CCI), was calculated (12).

Histopathology. Endoscopically or surgically resected specimens were immediately fixed in $10 \%$ buffered formalin solution and subsequently stained with hematoxylin and eosin. Experienced gastrointestinal pathologists who were completely blinded to the endoscopic diagnosis evaluated all the pathological specimens. Histological diagnoses were classified into 3 categories, namely, hyperplastic polyps, adenomas and carcinomas, including intramucosal and submucosal invasive types of carcinoma.

Statistical analysis. Mann-Whitney U-test, Pearson's correlation test, and Spearman's correlation test were used as appropriate. Receiver-operating-curve (ROC) analysis was used to determine the optimal cut-off values for assessing the colorectal lesions. For all studies, $\mathrm{p}$-values of $<0.05$ were set to determine the statistical significance. Statistical analysis was performed using the SPSS, version 11.5, software (SPSS Inc., Chicago, Ill, USA).

\section{Results}

A total of 54 colorectal mucosal lesions were detected, including 11 hyperplastic polyps, 30 adenomas and 13 carcinomas (8 intramucosal invasive types and 5 submucosal invasive types). The clinicopathological features of these lesions are presented in Table I. To quantify the AF intensity, the CCI was determined for each lesion after adjustments for the surrounding mucosal values. Fig. 1 shows the CCI values according to the size, histology and shape of the lesions. Interestingly, the CCI was significantly correlated with the size of the lesions $(\mathrm{r}=0.530, \mathrm{p}<0.0001)$ (Fig. 1A). With regards to the histology, the CCI values for carcinomas were significantly higher than those for adenomas $(\mathrm{p}=0.0001)$ or hyperplastic polyps $(\mathrm{p}<0.0001)$. In turn, the values for adenomas were significantly higher than those for hyperplastic polyps $(\mathrm{p}=0.0003)$ (Fig. 1B). On the other hand, the CCI was not associated with the lesion shape as assessed by macroscopic observation (16) (Fig. 1C).

Because CCI was associated with both the histology and size of the lesions, a further analysis was conducted to examine the relationship between the CCI and size of the lesions according to the histology. The results revealed that while the CCI was significantly correlated with the size of the lesion in the case of hyperplastic polyps $(r=0.641, p=0.0336)$ (Fig. 2), no correlation between the size and CCI was observed in the case of adenomas $(r=0.265, p=0.1574)$ or carcinomas $(r=0.174$, $\mathrm{p}=0.5699$ ), which showed consistently high values, irrespective of the size. In addition, the CCI was not associated with the depth of invasion in the carcinoma lesions [median CCI values, the intramucosal invasive type, $41.1(n=8)$; the submucosal invasive type, $30.0(n=5)]$.

Next we investigated the relationship between the CCI and histology of the lesions stratified into three different 
Table I. Clinicopathological features of the colorectal lesions.

\begin{tabular}{|c|c|c|c|c|c|c|c|c|c|}
\hline \multirow[b]{2}{*}{ Histology } & \multirow[b]{2}{*}{$\mathrm{n}$} & \multicolumn{2}{|c|}{ Macroscopic classification $^{\mathrm{a}}$} & \multicolumn{6}{|c|}{ Location } \\
\hline & & Flat elevated & Protruded & $\mathrm{C}$ & A & $\mathrm{T}$ & $\mathrm{D}$ & $\mathrm{S}$ & $\mathrm{R}$ \\
\hline Hyperplastic polyp & 11 & 10 & 1 & 1 & 1 & 3 & 0 & 3 & 3 \\
\hline Adenoma & 30 & 18 & 12 & 4 & 5 & 10 & 0 & 5 & 6 \\
\hline Carcinoma & 13 & 4 & 9 & 1 & 1 & 2 & 0 & 0 & 9 \\
\hline $\mathrm{n}$ & 54 & 28 & 22 & 6 & 7 & 15 & 0 & 8 & 18 \\
\hline
\end{tabular}

${ }^{\mathrm{a} B}$ Based on the Paris endoscopic classification of superficial neoplastic lesions (16). C, cecum; A, ascending colon; T, transverse colon; D, descending colon; S, sigmoid colon; R, rectum.

(A) Size

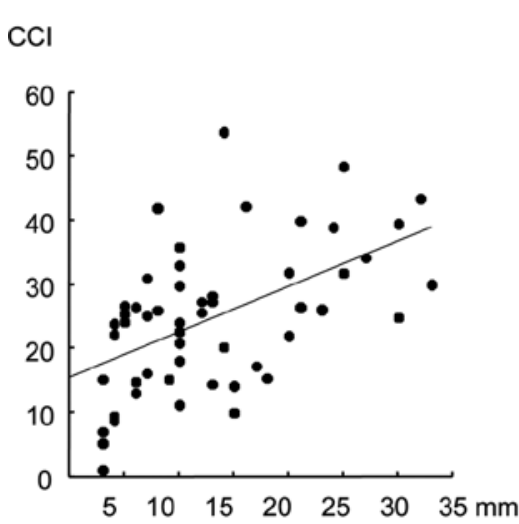

(B) Histology

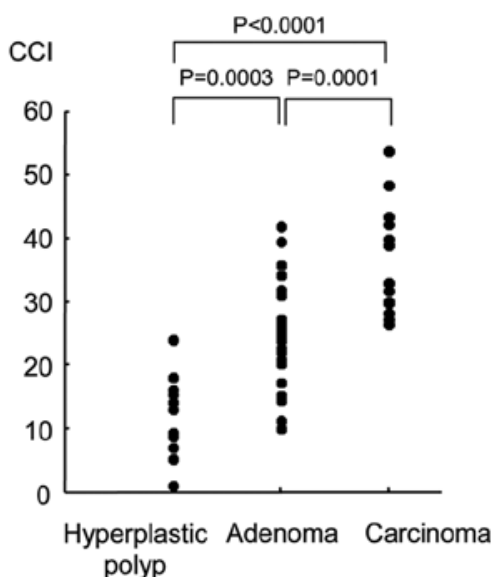

(C) Shape

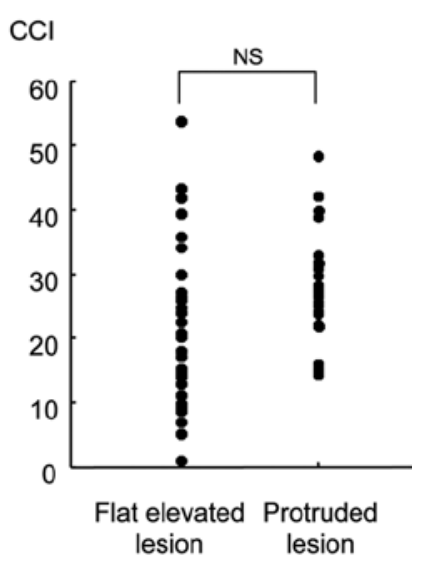

Figure 1. AF intensity as assessed by estimation of the color contrast index (CCI) in 54 colorectal mucosal lesions according to the size (A), histology (B) and shape $(\mathrm{C})$ of the lesion.

(A) Hyperplastic polyp

$\mathrm{CCl}$

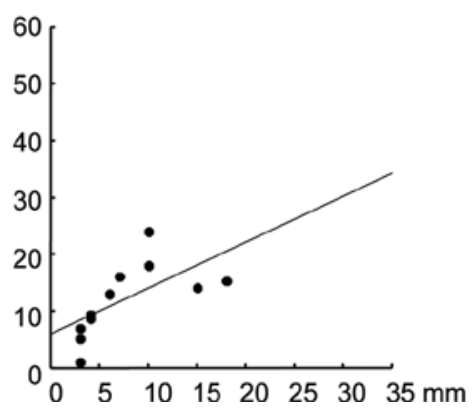

(B) Adenoma

$\mathrm{CCl}$

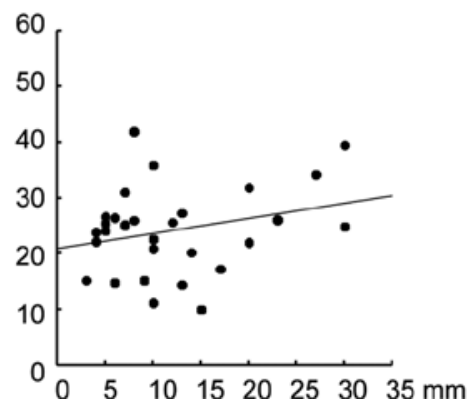

(C) Carcinoma

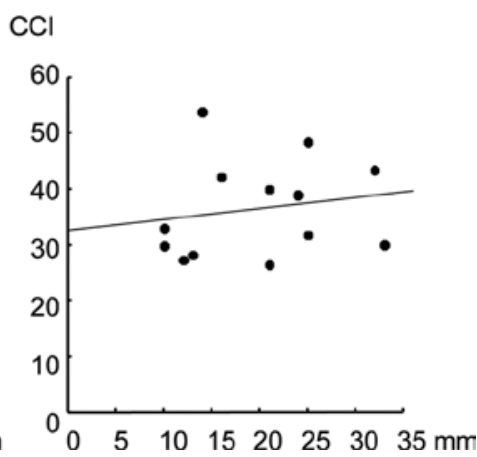

Figure 2. Relationship between the color contrast index (CCI) and size of colorectal hyperplastic polyps $(\mathrm{n}=11)(\mathrm{A})$, adenomas $(\mathrm{n}=30)(\mathrm{B})$ and carcinomas $(n=13)(C)$.

size groups (Fig. 3). It should be noted that the CCI values increased as the malignant potential of the lesions increased (that is, in the order of hyperplastic polyps $\rightarrow$ adenomas $\rightarrow$ carcinomas), irrespective of the lesion size $(r=0.797, p<0.0001$ for size $\leq 8 \mathrm{~mm}$; $\mathrm{r}=0.622, \mathrm{p}=0.0045$ for size $>8 \mathrm{~mm}$ but $\leq 15 \mathrm{~mm}$; $\mathrm{r}=0.644, \mathrm{p}=0.0071$ for size $>15 \mathrm{~mm}$ ). In each size group, the CCI tended to be higher for carcinomas than for adenomas, and also higher for adenomas than for hyperplastic 
(A) size $\leq 8 \mathrm{~mm}$

$\mathrm{CCl}$

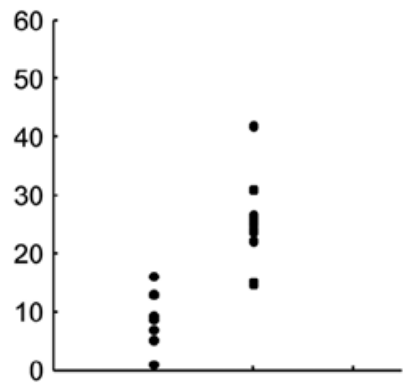

Hyperplastic Adenoma Carcinoma polyp
(B) $8 \mathrm{~mm}<$ size $\leq 15 \mathrm{~mm}$

$\mathrm{CCl}$

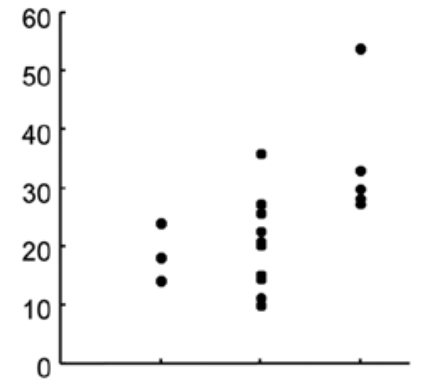

Hyperplastic Adenoma Carcinoma polyp
(C) $15 \mathrm{~mm}<$ size

$\mathrm{CCl}$

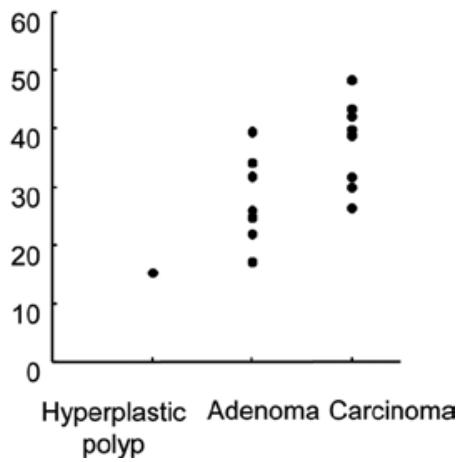

Figure 3. Relationship between color contrast index (CCI) and the histology of colorectal mucosal lesions classified according to the lesion size.

\section{A. Hyperplastic polyp}

B. Adenoma

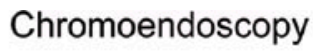

AF endoscopy
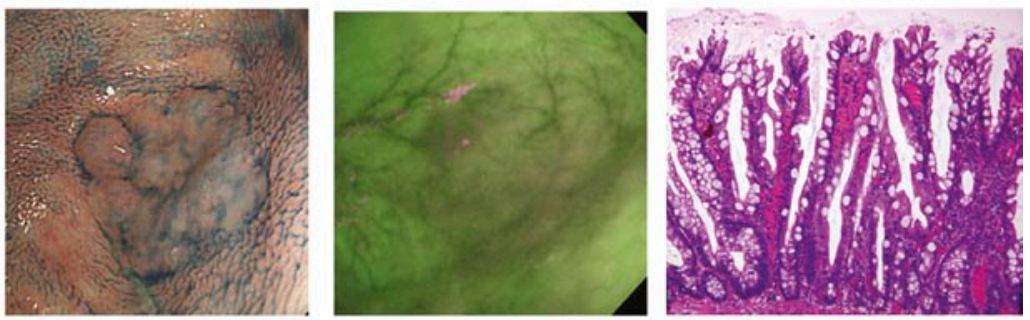

C. Carcinoma
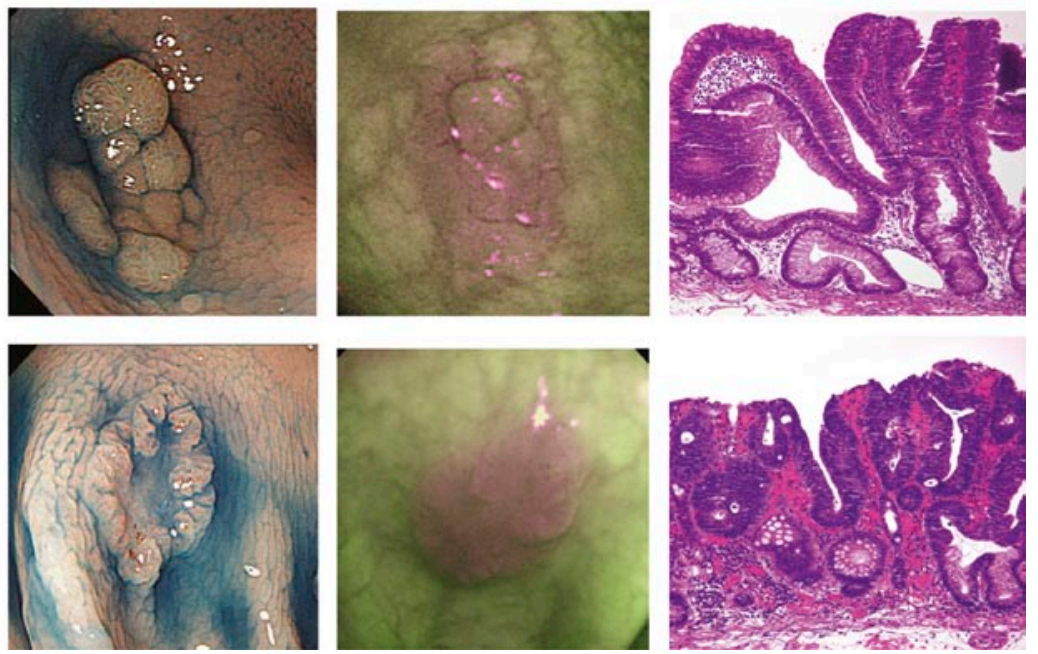

Figure 4. Representative chromoendoscopy, AF endoscopy and histological findings of a colorectal hyperplastic polyp (A), adenoma (B) and carcinoma (C).

polyps. Fig. 4 shows the representative pictures of chromoendoscopy, AF endoscopy, and histology of hyperplastic polyps (A), adenomas (B) and carcinomas (C).

Based on the differences in the CCI levels found among the groups, we calculated the efficacy of CCI in the differential diagnosis among the groups. The cut-off value was determined by the ROC curves as the value at the point showing the best sensitivity and specificity. The diagnostic accuracy of CCI for differentiating among colorectal lesions is shown in Table II. When an empirical cut-off value of 14.5 was used, AF endoscopy showed a sensitivity of $95.3 \%$ (41 of 43 lesions) and specificity of $63.6 \%$ (7 of 11 lesions) for distinguishing adenomas/carcinomas (neoplastic lesions) from hyperplastic polyps (non-neoplastic lesions). When the cut-off value of 28.0 was used, AF endoscopy showed a sensitivity of $84.6 \%$ (11 of 13 lesions) and specificity of $80.0 \%$ (24 of 30 lesions) for distinguishing between carcinomas and adenomas.

\section{Discussion}

Recently, several new endoscopic tools that enhance the detection and discrimination of mucosal lesions have been developed to 
Table II. Diagnostic accuracy of the color-contrast index.

\begin{tabular}{|c|c|c|c|c|c|}
\hline & Cut-off value & Sensitivity (\%) & Specificity (\%) & PPV (\%) & NPV $(\%)$ \\
\hline $\begin{array}{l}\text { Carcinoma/adenoma } \\
\text { vs. hyperplastic polyp }\end{array}$ & 14.5 & $\begin{array}{c}95.3 \\
(41 / 43)\end{array}$ & $\begin{array}{c}63.6 \\
(7 / 11)\end{array}$ & $\begin{array}{c}91.1 \\
(41 / 45)\end{array}$ & $\begin{array}{l}77.7 \\
(7 / 9)\end{array}$ \\
\hline $\begin{array}{l}\text { Carcinoma } \\
\text { vs. adenoma }\end{array}$ & 28.0 & $\begin{array}{c}84.6 \\
(11 / 13)\end{array}$ & $\begin{array}{c}80 \\
(24 / 30)\end{array}$ & $\begin{array}{c}64.7 \\
(11 / 17)\end{array}$ & $\begin{array}{c}92.3 \\
(24 / 26)\end{array}$ \\
\hline
\end{tabular}

PPV, positive predictive value; NPV, negative predictive value.

improve upon the accuracy afforded by conventional white-light endoscopy for the differential diagnosis of colorectal lesions (1). In this pilot study, we evaluated whether quantitative determination of the AF intensity might allow differentiation among hyperplastic polyps, adenomas and carcinomas.

Using the CCI, we clarified the clinicopathological factors responsible for the spectral differences in each lesion, and demonstrated that the size and histology are major determinants of the CCI. This implies that we should evaluate the association between CCI and histology taking into consideration the lesion size. In this study, we demonstrated that carcinomas showed high CCI values, irrespective of the lesion size. CCI allowed discrimination between carcinomas and adenomas with a satisfactorily good probability (sensitivity $84.6 \%$, specificity $80.0 \%$ ). Therefore, quantitative analysis using CCI can be potentially useful for distinguishing carcinomas from other lesions. However, large-scale studies are needed to accurately evaluate the usefulness of CCI for the diagnosis of colorectal carcinomas.

At present, the mechanisms responsible for the spectral differences among colorectal carcinomas are unclear. We could not find any association between the CCI values and the size, shape or depth of invasion in the carcinoma group. It is now suggested that the composition and bio-distribution of fluorophores, such as collagen, porphyrin, nicotinamide adenine dinucleotide hydrogenase (NADH), flavin adenine dinucleotide or lysosome granules, in the colorectal wall could be responsible for the differences (17-21). Metabolic alterations, inflammatory changes, hemoglobin content and absorption by the blood may also modify the AF intensity $(19,22)$. Investigation of the microscopic origins of tissue AF may provide further insight into the morphological structures and biochemical components of tissues, which is vital for improving the implementation of the AF technique for noninvasive in vivo tissue diagnostics.

Quantitative determination of the AF intensity was also helpful in the distinction between adenomas and hyperplastic polyps, which is in agreement with previous reports (11-15). McCallum et al have reported that using a different index of AF intensity (AF intensity ratio) allowed colorectal adenomas to be distinguished from hyperplastic polyps with a sensitivity of $85 \%$ and specificity of $81 \%$ (14). In this study, we showed higher CCI values for adenomas than for hyperplastic polyps. Furthermore, we could discriminate between colorectal neoplastic lesions (adenomas and carcinomas) and nonneoplastic lesions (hyperplastic polyps) with a satisfactorily high probability (sensitivity $95.3 \%$, specificity $63.6 \%$ ). These data indicate that CCI may be used to distinguish adenomas from hyperplastic polyps.

Although our results show promise for this new technology, one of the limitations of this study was that it was a single-center analysis of exclusively Japanese patients with a retrospective design, therefore, the results cannot be simply generalized to other institutes or other ethnic groups. Thus, the obvious next step is to evaluate this new technology in diverse populations and ethnic groups. Furthermore, the diagnosis in the study subjects in this study had been established. The question of the value of CCI in the diagnosis of colorectal lesions may be more appropriately answered in the context of a prospective study of patients conducted from the time of endoscopic detection of the lesions. In addition, the image resolution is still obscure and inadequate in AF endoscopy as compared with that in conventional white-light endoscopy.

In conclusion, these results suggest that quantitative analysis of the AF intensity using the CCI is helpful in discriminating among different types of colorectal mucosal lesions, including carcinomas. Large-scale, prospective studies are needed to evaluate the efficiency of differential diagnosis among the lesions by AF endoscopy.

\section{References}

1. Basu S, Torigian D and Alavi A: The role of modern molecular imaging techniques in gastroenterology. Gastroenterology 135: 1055-1061, 2008

2. Axelrad AM, Fleischer DE, Geller AJ, et al: High-resolution chromoendoscopy for the diagnosis of diminutive colon polyps: implications for colon cancer screening. Gastroenterology 110: 1253-1258, 1996.

3. Fu KI, Sano Y, Kato S, et al: Chromoendoscopy using indigo carmine dye spraying with magnifying observation is the most reliable method for differential diagnosis between non-neoplastic and neoplastic colorectal lesions: a prospective study. Endoscopy 36: 1089-1093, 2004.

4. Sano Y, Muto M, Tajiri H, Ohtsu A and Yoshida S: Optical/digital chromoendoscopy during colonoscopy using narrow-band image system. Dig Endosc 17: 43-48, 2005.

5. Chiu HM, Chang CY, Chen CC, et al: A prospective comparative study of narrow-band imaging, chromoendoscopy, and conventional colonoscopy in the diagnosis of colorectal neoplasia. Gut 56: 373-379, 2007.

6. Takehana S, Kaneko M and Mizuno H: Endoscopic diagnostic system using autofluorescence. Diagn Ther Endosc 5: 59-63, 1999.

7. Panjehpour M, Overholt BF, Vo-Dinh T, Haggitt RC, Edwards DH and Buckley FP 3rd: Endoscopic fluorescence detection of highgrade dysplasia in Barrett's esophagus. Gastroenterology 111: 93-101, 1996.

8. Mayinger B, Neidhardt S, Reh H, Martus P and Hahn EG: Fluorescence induced with 5-aminolevulinic acid for the endoscopic detection and follow-up of esophageal lesions. Gastrointest Endosc 54: 572-578, 2001. 
9. Kobayashi M, Tajiri H, Seike E, et al: Detection of early gastric cancer by a real-time autofluorescence imaging system. Cancer Lett 165: 155-159, 2001

10. Ohkawa A, Miwa H, Namihisa A, et al: Diagnostic performance of light-induced fluorescence endoscopy for gastric neoplasms. Endoscopy 36: 515-521, 2004.

11. Nakaniwa N, Namihisa A, Ogihara T, et al: Newly developed autofluorescence imaging videoscope system for the detection of colonic neoplasms. Dig Endosc 17: 235-240, 2005.

12. Aihara H, Sumiyama K, Saito S, Tajiri H and Ikegami M: Numerical analysis of the autofluorescence intensity of neoplastic and non-neoplastic colorectal lesions by using a novel videoendoscopy system. Gastrointest Endosc 69: 726-733, 2009.

13. Matsuda T, Saito Y, Fu KI, et al: Does autofluorescence imaging videoendoscopy system improve the colonoscopic polyp detection rate? - a pilot study. Am J Gastroenterol 103: 1926-1932, 2008.

14. McCallum AL, Jenkins JT, Gillen D and Molloy RG: Evaluation of autofluorescence colonoscopy for the detection and diagnosis of colonic polyps. Gastrointest Endosc 68: 283-290, 2008.

15. Mayinger B, Jordan M, Horner P, et al: Endoscopic light-induced autofluorescence spectroscopy for the diagnosis of colorectal cancer and adenoma. J Photochem Photobiol B 70: 13-20, 2003.
16. The Paris endoscopic classification of superficial neoplastic lesions: esophagus, stomach, and colon: November 30 to December 1, 2002. Gastrointest Endosc 58: S3-S43, 2003.

17. Sackmann M: Fluorescence diagnosis in GI endoscopy. Endoscopy 32: 977-985, 2000.

18. Romer TJ, Fitzmaurice M, Cothren RM, et al: Laser-induced fluorescence microscopy of normal colon and dysplasia in colonic adenomas: implications for spectroscopic diagnosis. Am J Gastroenterol 90: 81-87, 1995.

19. Zonios GI, Cothren RM, Arendt JT, et al: Morphological model of human colon tissue fluorescence. IEEE Trans Biomed Eng 43: 113-122, 1996.

20. Moesta KT, Ebert B, Handke T, et al: Protoporphyrin IX occurs naturally in colorectal cancers and their metastases. Cancer Res 61: 991-999, 2001.

21. Pradhan A, Pal P, Durocher G, et al: Steady state and timeresolved fluorescence properties of metastatic and non-metastatic malignant cells from different species. J Photochem Photobiol B 31: 101-112, 1995.

22. Marchesini R, Pignoli E, Tomatis S, et al: Ex vivo optical properties of human colon tissue. Lasers Surg Med 15: 351-357, 1994. 